This is a self-archiving document (manuscript version):

Analysis of solar cell cross sections with micro-light beam induced current $(\mu \mathrm{LBIC})$

By: Breitwieser, Matthias; Heinz, Friedemann D.; Büchler, Andreas;

Kasemann, Martin; Schön, Jonas; Warta, Wilhelm; Schubert, Martin C.

Published in: Solar Energy Materials and Solar Cells

Volume 131, December 2014, Pages 124-128 (Please be aware: Page numbering in this manuscript can be different to published version!)

Publisher: Elsevier | Year: 2014

DOI: 10.1016/j.solmat.2014.05.002

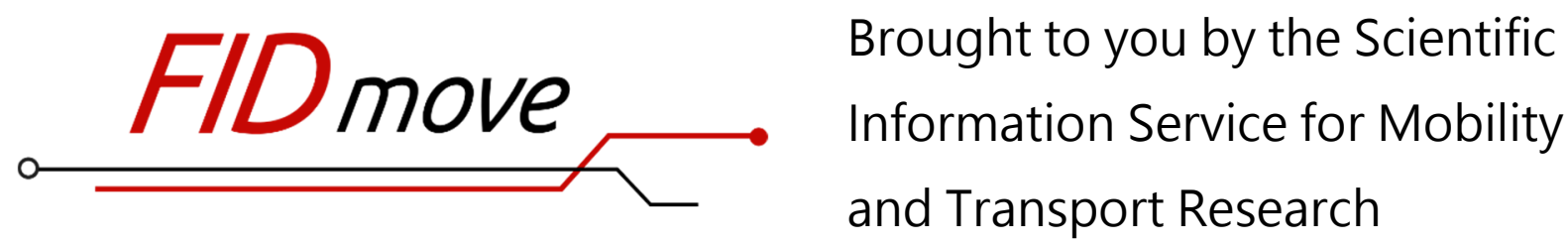

Fachinformationsdienst Mobilitäts- und Verkehrsforschung

Website: www.fid-move.de

Repository: publish.fid-move.de

Contact: publish@fid-move.de

(C) 2020. This manuscript version is made available under the CC-BYNC-ND 4.0 license.

http://creativecommons.org/licenses/by-nc-nd/4.0/ 


\title{
Analysis of solar cell cross sections with micro-light beam induced current ( $\mu$ LBIC)
}

\author{
Matthias Breitwieser ${ }^{\mathrm{a}, *}$, Friedemann D. Heinz ${ }^{\mathrm{a}}$, Andreas Büchler ${ }^{\mathrm{a}}$, Martin Kasemann ${ }^{\mathrm{b}}$, \\ Jonas Schon ${ }^{a}$, Wilhelm Warta ${ }^{a}$, Martin C. Schubert ${ }^{a}$ \\ ${ }^{\text {a }}$ Fraunhofer Institute for Solar Energy Systems, Heidenhofstr. 2, 79110 Freiburg, Germany \\ ${ }^{\mathrm{b}}$ University of Freiburg, Department of Microsystems Engineering,Georges-Koehler-Allee 106, 79110 Freiburg, Germany
}

\section{A B S T R A C T}

\begin{abstract}
A highly resolving micro-light beam-induced current ( $\mu$ LBIC)-system is presented in this work. Based on the laser excitation via an optical microscope, current values can be measured with sub-micron precision. We show, that this non-destructive, light-based approach delivers superior results to a reference electron microscope based electron beam induced current method concerning contrast and robustness towards reflection differences, whereas no vacuum is needed, no charging effects can occur and equal resolution is achieved. $\mu$ LBIC allows therefore mapping of pn-junctions at silicon solar cell cross sections. By combination of $\mu$ LBIC with other measurement methods in the same setup, such as micro-Raman spectroscopy, complementary microscopic information about material stress or crystallinity and electronic properties at the same region of interest on the sample is revealed. By applying $\mu$ LBIC for analyzing silicon solar cross sections, two characterization examples of current technological relevance are presented: enhanced dopant diffusion along grain boundaries between grains with different orientations is quantified and the impact of a nickel silicide spike on local charge collection quality is studied.
\end{abstract}

\section{Introduction}

For the microscopic characterization of charge- and currentrelated features of local back surface fields, selective emitters, or electrical properties like contact formation or local processing defects on a microscopic scale, reliable and highly resolving characterization methods are required. Due to the size of the analyzed structures in the micrometer range, electron microscopes are commonly used. Functional analysis of these structures became possible with the development of the electron beam induced current method (EBIC) [1]. An alternative approach for micro-characterization tasks uses the confocal laser scanning microscope, introduced by Minsky et al. [2]. This microscope works under atmospheric pressure, does not show any charging effects and is fully non-destructive. By extending the setup in order to detect backscattered and emitted luminescent light, it can be used in several more advanced configurations: MicroPhotoluminescence Spectroscopy ( $\mu$-PLS) delivers lifetime information of photovoltaic material with sub-micron resolution $[3,4]$.

\footnotetext{
* Corresponding author. Tel.: + 49 761/4588 5321; fax: +49 761/4588 9250

E-mail address: matthias.breitwieser@ise.fraunhofer.de (M. Breitwieser).
} 
Micro-Raman spectroscopy ( $\mu$-RS) reveals data about the crystallinity or material stress or doping density of a sample $[5,6]$ with a diffraction limited spatial resolution of $200 \mathrm{~nm}$.

Our combined confocal $\mu \mathrm{PLS} / \mu \mathrm{RS}$ setup has been enhanced by a micro-light beam induced current ( $\mu$ LBIC) setup by contacting the sample to a suitable low-noise current amplifier. A microscopic $\mu$ LBIC-system has first been introduced by De Vittorio in 1999, who applied the technique to direct semiconductor structures [7].

In this work we present results obtained with a high-resolution multifunctional $\mu \mathrm{LBIC}, \mu \mathrm{RS}$ and $\mu$ PLS-tool and application to the characterization of grain boundaries and nickel silicide spikes. Fig. 1 shows a schematic of the setup. Compared to published configurations $[7,8]$, the higher resolution of the presented setup enables the examination of polished cross sections of silicon solar cells with $\mu$ LBIC. The focussed laser excitation leads to an extractable photocurrent in the space charge region of a pn-junction or a Schottky barrier. This enables the measurement of junction depths or the localization of inhomogeneities like crystal defects or damages in these layers with sub-micron resolution. Via the same laser excitation for $\mu \mathrm{RS}$ and the $\mu$ LBIC-measurement, two spatially corresponding 2D-mappings are obtained. Electrical information from the $\mu \mathrm{LBIC}$ measurement and material information from $\mu$ RS delivers additional quantitative information about material stress or the crystal orientation. 


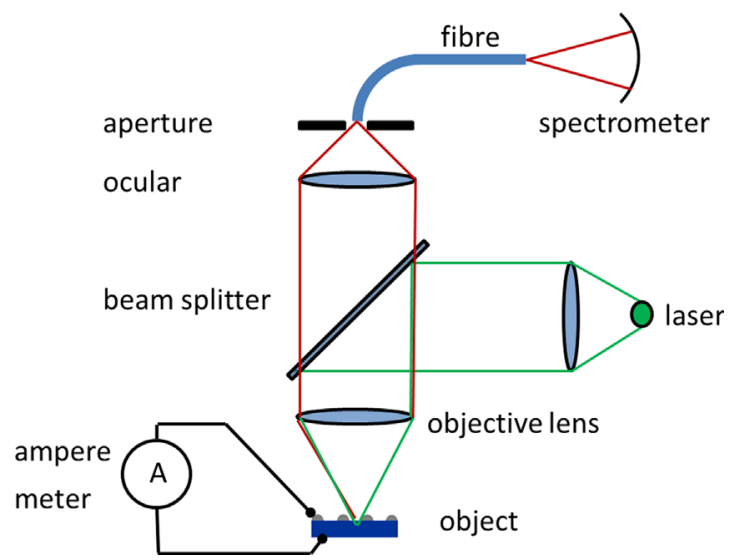

Fig. 1. Schematic of the $\mu \mathrm{LBIC} / \mu \mathrm{RS} / \mu \mathrm{PLS}$ setup.

\section{Measurement principle}

Our setup is based on a confocal laser scanning microscope, where the examined sample is illuminated by focused laser light (Nd:YAG) with the wavelengths of $355 \mathrm{~nm}$ or $532 \mathrm{~nm}$. To scan the region of interest on the sample, the motorized scanning stage is moved in a $x-y$ direction with a precision of below $100 \mathrm{~nm}$.

The measurement principle of $\mu \mathrm{LBIC}$ is based on the local laser excitation of charge carriers in the illuminated piece of semiconductor. The size of the laser spot is given by the numerical aperture of the microscope objective lens and by the wavelength of the laser and is diffraction limited. The absorption profile can be described with the exponential decay of light in material according to Lambert-Beer:

$I(z)=I_{\mathrm{ph}} \cdot e^{-\alpha z}$

$\alpha$ is the absorption coefficient and $I_{\mathrm{ph}}$ the initial light intensity at the sample surface. In the presence of a pn-junction, generated charges are separated and an illumination dependent current can be measured. Via a 2D-scan, a map with light beam induced current values can be obtained. At a non-distorted site on a planar device, without locally increased SRH-recombination, the current $I_{0}$ as given in formula (2) is measured, determined by the background carrier lifetime in the material [9]. Note that Eq. (2) holds only for a planar device with surface carrier collection. $D_{n}$ is the diffusion constant of the carriers

$I_{0} \propto q \cdot \int_{0}^{\infty} \alpha \cdot e^{-\alpha z} \cdot e^{-z / \sqrt{D_{n} \tau}} d z$

In the direct surrounding of recombination active regions, the extractable current drops significantly, since locally recombination active structures, like precipitates, grain boundaries (GBs) or dislocation clusters, reduce the induced carrier density $\Delta n$, and thus the $\mu$ LBIC-signal. This locally induced current $I^{*}$ is affected by the amount of the local recombination activity in the illuminated area.

This difference between $I_{0}$ and the locally reduced $I^{*}$ leads to a contrast $K$ between areas with locally increased recombination and undistorted sites, which can be expressed using

$K=\frac{I_{0}-I^{*}(x)}{I_{0}}$

In the cross section configuration, the generation of the extractable induced current is dependent on the distance between the confocal laser spot and a charge separating layer, such as a pn-junction or a Schottky barrier. The induced current peaks at the pn-junction position and decays in both directions according to 
the diffusion length, cross section surface, and SRH recombination in the two layers.

\section{Results}

\subsection{Comparison of $\mu \mathrm{LBIC}$ and EBIC}

To verify sensitivity and spatial resolution of the introduced $\mu$ LBIC technique, a small angle grain boundary on a multicrystalline solar cell with very low recombination activity is chosen. As reference we use our EBIC tool (Hitachi SU-70, Gatan SmartEBIC). In the case of $\mu$ LBIC electron-hole pairs are generated effectively by the $532 \mathrm{~nm}$ light; the collected current is determined by SRH-, surface-, Auger recombination, the local charge collection efficiency and the carrier mobility. The lateral extension of the laser spot of around $500 \mathrm{~nm}$ and the low light absorption depth of $1 \mu \mathrm{m}$ leads to a small excitation volume and therefore high injection density. In the high injection regime dominating Auger recombination restricts in turn the size of the diffusion region to typically below $1 \mu \mathrm{m}$ [3]. For the case of EBIC and an adequate acceleration voltage of $15 \mathrm{keV}$, suitable for resolving structures with very low recombination activity, the approximately spherical excitation volume is around $4 \mu \mathrm{m}$ in diameter.

Fig. 2 shows a EBIC (a) and $\mu$ LBIC (b) measurement at the same spot at a small angle GB on a multicrystalline silicon solar cell, which was intentionally chosen to exhibit only low recombination contrast. Obviously the $\mu$ LBIC-measurement delivers equal resolution and increased contrast at the GB (indicated in yellow), and is less influenced by the anisotropic texture of the solar cell surface (region marked with red dashed line), compared to EBIC. The $\mu$ LBIC line scan in the diagram (Fig. 2c) achieves a 2.5 times higher contrast and equal resolution at the GB. The same current amplifier (Stanford Research Systems SR-570) and sample holder were used to ensure equal measurement conditions.

The larger generation volume of EBIC is due to scattering of the electrons in the material and explains the lower contrast values in the current mapping compared to the $\mu$ LBIC case at microscopic structures with low recombination activity. Furthermore the enhanced scattering of the electron beam at microstructured surfaces leads to a stronger influence of the surface texture on the EBIC-measurement. This is a considerable drawback, since solar cells are typically textured to reduce reflection losses. The coupling of laser light into the sample is less affected by the texture. Despite the diffraction limited excitation spot size of $\mu \mathrm{LBIC}$, the spatial resolution is competitive to EBIC, since the spatial resolution of both methods is given by the local diffusion length of the excited carriers.

\subsection{Resolving grain boundary diffusion at multicrystalline solar cells}

An example to illustrate the performance of this characterization method is the analysis of the diffusion characteristics of grain boundaries in multicrystalline solar cells. The investigated sample is a fully processed standard mc solar cell. We examine a polished cross section cut from this sample. The cross sections were produced by mechanical polishing with a commercial polisher with subsequently decreased polish particle size.

Diffusion of impurities was reported to be enhanced along certain GBs in metals and in semiconductors like silicon [10-13]. For dopant atoms such as phosphorous or boron, tracing of the grain boundary diffusion path is easily accessible, since a pnjunction is formed along the GB, if diffusion of the dopants takes place. Up to now, only electron beam induced current (EBIC) measurements were performed to image these so-called "pn-spikes" or "pipe-diffusion" $[10,11,13]$. We use here the 

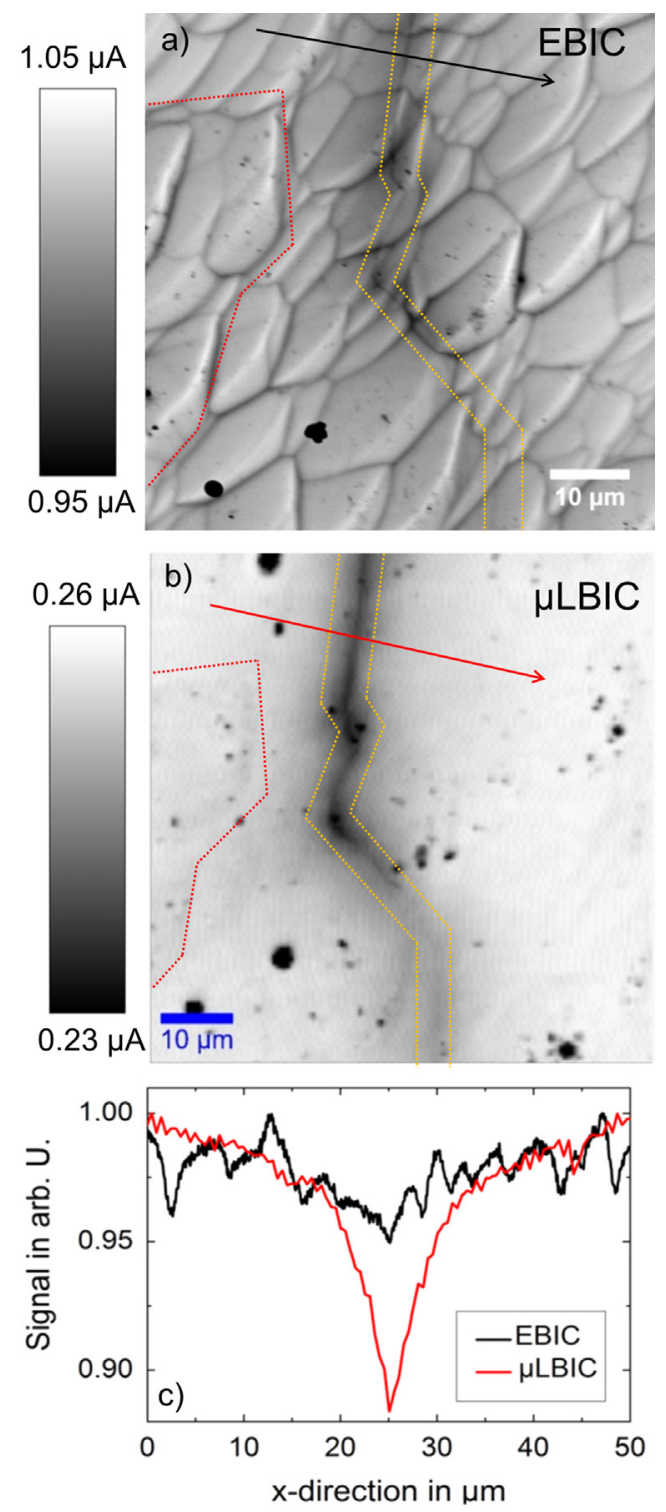

Fig. 2. Comparison between EBIC (a) $\mu$ LBIC (b) and at a small angle grain boundary in a multicrystalline solar cell. The position of the GB is depicted with yellow dashed lines. Only a minor influence of the texture on the $\mu$ LBIC-measurement is observed (see regions marked with red dashed line). The black and red arrows correspond to the line scans, depicted in the diagramm (c). The black spots correspond to surface contamination on the solar cell surface and therefore shading of the excitation beam. (For interpretation of the references to color in this figure legend, the reader is referred to the web version of this article.)

combination of $\mu$ RS and $\mu$ LBIC to analyze this phenomenon. The advantages of our light based method are that the injection density can be controlled by a simple laser power measurement and subsequent microscopic lifetime mapping is possible with $\mu$ PLS within the same setup.

The sketches in Fig. 3 ( $c$ and $\mathrm{f}$ ) explain the sample configuration. The $\mu$ RS-measurements shown in Fig. 3 (a and d) deliver an intensity contrast between the different grain orientations and thus indicate the position of the GB. The $\mu$ LBIC-mapping of the grain boundary (Fig. 3 b) shows the pn-junction parallel to the substrate surface and a sharp space charge region exactly along the GB. An electron backscatter diffraction (EBSD)-measurement identifies the grain boundary type with enhanced diffusion as $\Sigma 3$. An enhanced $\mu$ LBIC signal was also measured at five other $\Sigma 3$ grain boundaries on the multicrystalline sample, but with deviating $\mu$ LBIC signal depths between 5 and $18 \mu \mathrm{m}$ (see Table 1). Based on 


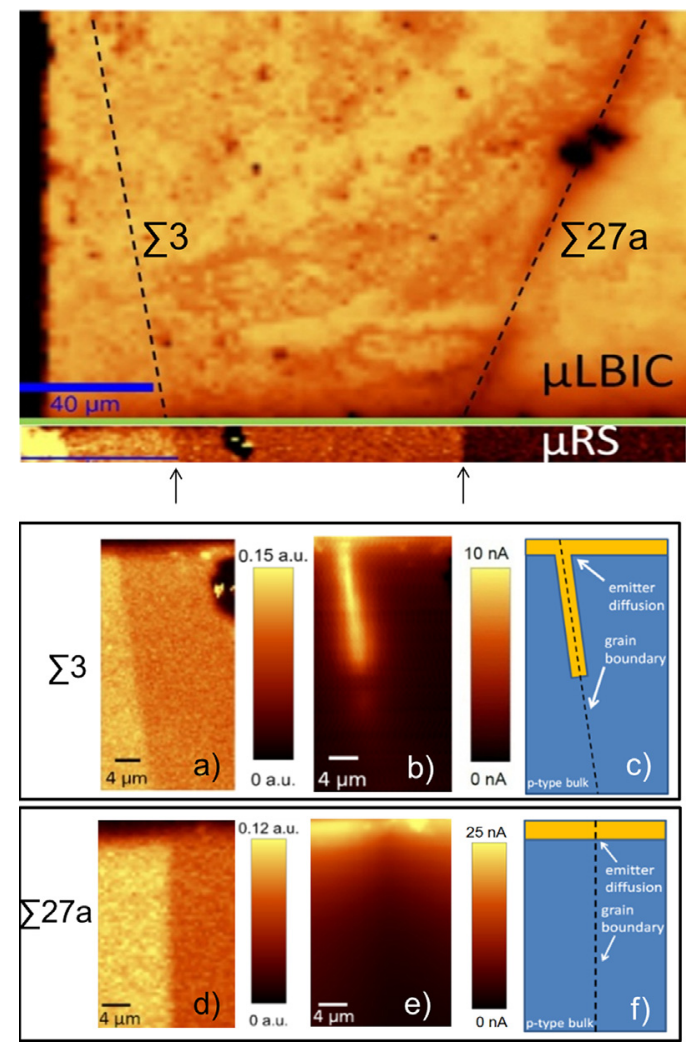

Fig. 3. Two grain boundaries with different $\mu$ LBIC characteristics are shown: with electron backscattered diffraction (EBSD) the type of the GBs is determined to $\Sigma 3$ and $\Sigma 27$ a. High resolution $\mu$ RS measurements $(\mathrm{a}, \mathrm{d})$ show the position of the two GBs. $\mu$ LBIC scans $(b, e)$ at the GBs in the cross section configuration reveal a $18 \mu \mathrm{m}$ deep space charge region along the $\Sigma 3 \mathrm{~GB}$, whereas the $\Sigma 27$ a GB shows a drop in the $\mu$ LBIC signal (left). The sample configuration is explained in the sketches in (c, f). An additional $\mu$ LBIC scan (upper image) from the top of the cell reveals an enhanced recombination activity at the $\Sigma 27$ a grain boundary. The green line corresponds to the sample edge. The overview cross-section- $\mu$ RS (upper image) signals the position of the two grain boundaries (black arrows). (For interpretation of the references to color in this figure legend, the reader is referred to the web version of this article.)

Table 1

Summary of the calculated diffusion constants of all measured grain boundaries on a multicrystalline solar cell cross section.

\begin{tabular}{llll}
\hline & Diffusion & $\begin{array}{l}\text { Calculated } \\
D_{\mathrm{GB}} / D_{\text {bulk }}\end{array}$ & $\begin{array}{l}\text { Enhanced } \\
\text { recombination? }\end{array}$ \\
\hline$\Sigma 3$ (shown in Fig. 1) & Yes & $2.7 \cdot 10^{4}$ & No \\
$\Sigma 3$ & Yes & $0.2 \cdot 10^{4}$ & No \\
$\Sigma 3$ & Yes & $1.8 \cdot 10^{4}$ & No \\
$\Sigma 3$ & Yes & $0.8 \cdot 10^{4}$ & No \\
$\Sigma 3$ & Yes & $0.3 \cdot 10^{4}$ & No \\
$\Sigma 3$ & Yes & $0.2 \cdot 10^{4}$ & No \\
$\Sigma 27 a$ (shown in Fig. 1) & No & - & Yes \\
Random large angle GB & No & - & Yes \\
Large angle GB (measured in & Yes & $0.8 \cdot 10^{4}$ & Not reported \\
$\quad[10]$ ) & & & \\
\hline
\end{tabular}

the model description of Harrison [14], the diffusion coefficient ratio between the bulk diffusion coefficient $D_{\text {bulk }}$ and the GBdiffusion coefficient $D_{\mathrm{GB}}$ can be calculated by extracting the GB diffusion depth of each GB directly from the $\mu$ LBIC-signal profile. $D_{\text {bulk }}=5 \cdot 10^{-16} \mathrm{~cm}^{2} \mathrm{~s}^{-1}$ is taken from [15] for phosphorous diffusion in silicon at the emitter diffusion temperature of $830{ }^{\circ} \mathrm{C}$. The different values for $D_{\mathrm{GB}} / D_{\text {bulk }}$ for the investigated $\Sigma 3 \mathrm{GBs}$, summarized in Table 1 are in the range of the value reported for a large angle grain boundary in the literature [10]. A second grain boundary of different types ( $\Sigma 27 \mathrm{a})$ is also investigated with $\mu$ LBIC 
(fig. 3 e), but no enhanced diffusion is found. An additional $\mu$ LBIC measurement from the top of the solar cell (Fig. 3, upper image) reveals that the recombination activity at the $\Sigma 3$ GB is not enhanced compared to the $\Sigma 27$ a GB, where the recombination activity is very high.

A possible explanation for our results could be the structural difference of $\Sigma 3$ and larger $\Sigma$ GBs: $\Sigma 3$ grain boundaries are known to show a low recombination activity, since they are scarcely decorated with impurities and have very little lattice mismatch. This characteristic could facilitate the observed pipe diffusion along these grain boundaries. GBs like $\Sigma 27$ a show considerably higher recombination activity which is related to an increased amount of impurity decoration. The decoration or the high stress might hinder the diffusion of additional dopant atoms along the GB-plane. These findings are in disagreement with [16], up to now the only classification of grain boundary dependent diffusion. In [16] diffusion is reported to take place only along other large angle grain boundaries than $\Sigma$ 3. These uncertainties motivate further investigation of the dopant diffusion along grain boundaries. Furthermore, especially for the development of high efficiency multicrystalline cells, precise and homogeneous pn-junctions are crucial.

\subsection{Investigating the microscopic impact of nickel silicide spikes}

Copper metallization is a cost-effective candidate to replace the expensive state-of-the-art use of silver $[17,18]$. But, since copper diffuses very fast in silicon and creates unwanted recombination activity, a protecting diffusion barrier must be realized between the silicon solar cell and the copper metallization. Nickel plating is one option to obtain a stable diffusion barrier [19]. In order to achieve good electrical and mechanical contact, the nickel layer must be annealed to enable the growth of nickel silicide. A main drawback is still the crucial role of this nickel silicide formation: On the one hand, the silicide formation is necessary for good contact adhesion, on the other hand silicide spikes in the space charge region were found to reduce severely the cell performance. As Büchler et al. have shown, these early break-down sites are caused by numerous local nickel silicide spikes [20]. They may induce local non-ohmic shunts and can cause an early break-down characteristic under reverse-bias [20,21]. With $\mu$ LBIC we can contribute to understanding the severe electrical influence of these spikes on a microscopic scale. The analyzed sample is an ion polished cross section of a fully processed mono crystalline solar cell with a plated nickel layer on the front side. The cross section was manufactured by ion polishing a breaking edge of the solar cell. After nickel plating the solar cell was annealed in forming gas atmosphere at $350{ }^{\circ} \mathrm{C}$ for $10 \mathrm{~min}$ to achieve nickel silicide formation and adhesion. Thereby, the pseudo-fill factor (pFF) of the cell dropped significantly from 0.82 to 0.5 . One microscopic spike (approximately $500 \mathrm{~nm}$ in diameter) was then localized and imaged with a SEM and subsequently analyzed with our confocal $\mu$ LBIC and $\mu$ RS tool. Fig. 4 a) shows the spatially correspondent overlay of a SEM image of the analyzed spike and a $\mu$ LBIC measurement, in Fig. 4 b), the region of interest of same SEM image is superimposed with a mapping of the spectral position of the scattered Raman signal peak, correlating with material stress.

As shown in Fig. 4 b), the thermal growth of the nickel silicide spike into the undistorted silicon lattice leads to material stress, which is detectable with $\mu \mathrm{RS}$ : at the tip of the spike tensile stress is generated, leading to a peak-shifted Raman signal (Fig. 4 b). In the surrounding of the nickel silicide the situation is inverted, the Raman signal is shifted towards higher energies, therefore the lattice constant decreases due to the compressive stress induced by the nickel silicide. The drop of the $\mu$ LBIC signal at the spike reveals the local damage of the electrical solar cell properties 


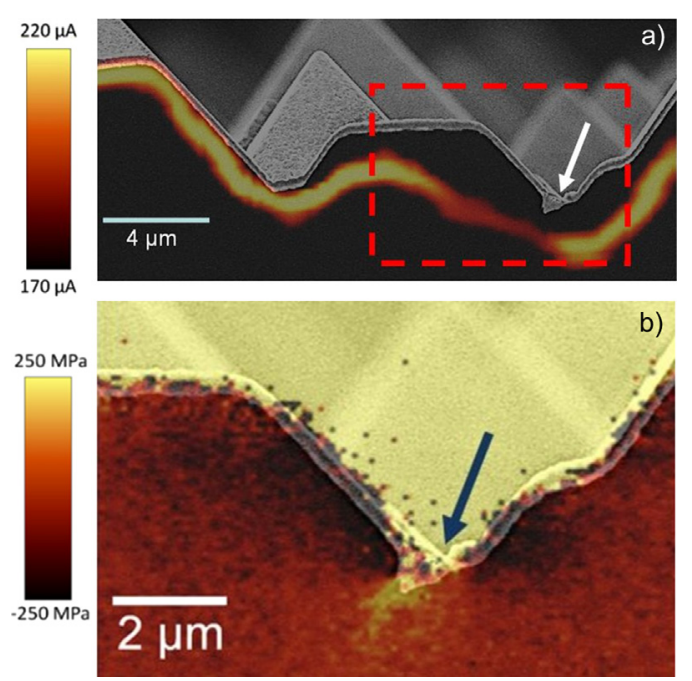

Fig. 4. In the upper image (a), the superposition of a SEM-image and a $\mu$ LBIC-map is shown. The $\mu$ LBIC-signal drops at the spike (white arrow), indicating the reduced charge collection in this region. The spectral peak-shift of the $\mu$ RS signal (b) reveals material stress at the spike in the MPa-range.

caused by the nickel silicide spike (Fig. 4 a). With these results, the observation of a drop in global cell performance can be related to microscopic material stress and the local decrease of the charge collection probability in the surrounding of the nickel silicide spike. In [21], annealing-induced deep nickel silicide structures were found to cause local breakdown sites that radiate visible light while breakdown occurs. The assumption, that likewise the electrical properties are severely degraded in the direct surrounding of a nickel silicide spike that penetrate the space charge region, is confirmed here. We demonstrate that the charge collection probability in the surrounding of the silicide spike is reduced within a dimension of about $4 \mu \mathrm{m}$.

\section{Conclusion}

The benefit of a multifunctional microscopic tool for advanced solar cell characterization is presented in this work. The nondestructive $\mu$ LBIC-method delivers superior results to EBIC concerning contrast and robustness towards reflection differences, whereas no vacuum is needed, no charging effects can occur and equal spatial resolution is achieved. In combination with $\mu R S$, microscopic information about the crystal orientation of material stress can be extracted with the same setup. Therefore, via simultaneous measurements, complementary material information can be gained without moving the sample. Cross sections of silicon solar cells are examined for the first time with a light based $\mu$ LBIC technique.

Two examples of current technologically relevant problems demonstrate clearly the versatility of the presented joint characterization with $\mu$ LBIC and $\mu$ RS: dopant diffusion along GB can now be analyzed with the multifunctional $\mu$ LBIC setup. Enhanced phosphorous diffusion is found at GBs with low recombination activity. An EBSD analysis reveals that these GBs are of $\Sigma 3$ type. These findings may indicate a possible correlation between GB recombination activity and dopant diffusion velocity, which is resolvable reliably with the combination of $\mu$ RS and $\mu$ LBIC.

Furthermore the impact of a microscopic nickel silicide spike in the space charge region is examined with $\mu$ LBIC and $\mu$ RS. Material stress and decrease of the charge collection probability occur at the silicide spike and harm the overall cell efficiency. A local drop 
of the electrical performance within a spatial extent of $4 \mu \mathrm{m}$ in the surrounding of a nickel silicide spike is resolved with $\mu$ LBIC.

Even if the excitation by the short wavelength light used is restricted by diffraction, this unique combination of $\mu \mathrm{PLS}, \mu \mathrm{RS}$ and $\mu$ LBIC enables a competitive performance compared to scanning electron methods.

\section{Acknowledgments}

The authors would like to thank the ISE PV-TEC and the clean room team for the processing support, Michaela Winterhalder and Johannes Hoerr for the cross-section preparation and Florian Schindler for the EBSD measurement support.

\section{References}

[1] H.J. Leamy, et al., Charge collection scanning electron microscopy, J. Appl. Phys. 53 (6) (1982) R51-R80.

[2] M. Minsky, et al., Memoir on inventing the confocal scanning microscope, Scanning 10 (4) (1988) 128-138.

[3] Paul Gundel, et al., Quantitative carrier lifetime measurement with micron resolution, J. Appl. Phys. 108 (3) (2010) 033705.

[4] Paul Gundel, et al., Micro-photoluminescence spectroscopy on metal precipitates in silicon, Phys. Status Solidi (RRL): Rapid Res. Lett. 3 (7-8) (2009) 230-232.

[5] F.D. Heinz, W. Warta, M.C. Schubert, Optimizing micro Raman and PL spectroscopy for solar cell technological assessment, Energy Procedia 27 (2012) 208

[6] M. Becker, et al., Highly p-doped regions in silicon solar cells quantitatively analyzed by small angle beveling and micro-Raman spectroscopy, J. Appl. Phys. 106 (7) (2009) 074515.

[7] M. De Vittorio, et al., Sub-micron photocurrent mapping of heterostructures by micro-probe optical-beam induced current, Rev. Sci. Instrum. 70 (8) (1999) 3429-3431.

[8] J. Martin, et al., A versatile computer-controlled high-resolution LBIC system, Prog. Photovolt.: Res. Appl. 12 (4) (2004) 283-295.

[9] J. Marek, et al., light-beam induced current characterization of grain boundaries, J. Appl. Phys. 55 (2) (1983) 318-326.

[10] A.D. Buonaquisti, et al., Diffusion characteristics of boron and phosphorus in polycrystalline silicon, Thin Solid Films 100 (3) (1983) 235-248.

[11] O. Krüger, et al., Interaction of crystal defects with pn junctions in multicrystalline Si solar cells, Solid State Phenom. 69 (1999) 173-178.

[12] Y. Mishin, Chr. Herzig, Grain boundary diffusion: recent progress and future research, Mater. Sci. Eng. A 260 (1) (1999) 55-71.

[13] Martin Kittler, Winfried Seifert, O. Krüger, Electrical behaviour of crystal defects in silicon solar cells, Solid State Phenom. 78 (2001) 39-48.

[14] L.G. Harrison, et al., Influence of dislocations on diffusion kinetics in solids with particular reference to the alkali halides, Trans. Faraday Soc. 57 (1961) 1191-1199.

[15] S.M. Sze, Semiconductor Devices: Physics and Technology, 2nd edition, Wiley, New York, NY, USA, 2002.

[16] H.J. Moller, Semiconductors for Solar Cells, Artech House, Boston, 1993.

[17] J. Bartsch, A. Mondon, K. Bayer, C. Schetter, M. Horteis, S.W. Glunz, Quick determination of copper-metallization long-term impact on silicon solar cells, J. Electrochem. Soc. 157 (10) (2010) H942-6.

[18] E.J. Lee, D.S. Kim, S.H. Lee, Ni/Cu metallization for low-cost high-efficiency PERC cells, Sol. Energy Mater. Sol. Cells 74 (1) (2002) 65-70.

[19] A., Knorz, et al.. Laser ablation of antireflection coatings for plated contacts yielding solar cell efficiencies above $20 \%$, in: Proceedings of the 24th European Photovoltaic Solar Energy Conference, Hamburg, Germany, 2009.

[20] Büchler, Andreas, et al., Localization and characterization of annealinginduced shunts in Ni-plated monocrystalline silicon solar cells, Phys. Status Solidi (RRL): Rapid Res. Lett. (2014).

[21] Sven Kluska, et al., Micro characterization of laser structured solar cells with plated Ni-Ag contacts, Sol. Energy Mater. Sol. Cells 120 (2014) 323-331. 